\title{
Are Dyslipidemia Following Obesity and Hyperglycemia as an Indicator of Diabetes Involved in Dental Caries Development in Zucker Rats?
}

Yasushi Kodama"\#, Kiyokazu Ozaki2\#, Tomoya Sano², Yui Terayama², Taiki Nishimoto", Yoshihiko Taniguchi' and Tetsuro Matsuura*

${ }^{1}$ Laboratory of Clinicopathological Therapeutics, Faculty of Pharmaceutical Sciences, Hiroshima International University, Hiroshima, Japan

${ }^{2}$ Department of Pathology, Faculty of Pharmaceutical Sciences, Setsunan University, Osaka, Japan

\#Contributed equally to this work

\begin{abstract}
Several studies indicated that both obesity and diabetes are related to dental caries development, but their involvement remains unclear. Thus, we investigated the effect of obesity and diabetes on dental caries development using obese Zucker fatty (ZF) rats, obese Zucker diabetic fatty (ZDF) rats, and Zucker lean (Lean) rats as controls.

Eight ZF, 6 ZDF, and 7 Lean male rats were studied. We macroscopically examined the mandibular and maxillary molars and evaluated soft X-ray photographs for caries development. From each group, 5 mandibles and maxillae were examined histologically.

The ZF rats' average body weight was more than twice that of the other 2 groups, but the ZDF and Lean rats had comparable body weights. The average triglyceride and total cholesterol levels were elevated in the ZF and ZDF rats, but the ZF rats' average triglyceride and total cholesterol levels were three times and twice, respectively, those of the ZDF rats. The ZDF rats became diabetic after 13 weeks of age and demonstrated a high blood glucose level $(>500 \mathrm{mg} / \mathrm{dL}$ ) after 23 weeks of age. The ZF rats had a significantly higher blood glucose level than that of Lean rats after 23 weeks of age but had about one-half that of the ZDF rats. The ZDF rats had significantly more molar caries and greater alveolar bone resorption than the other two groups; the ZF rats' caries and bone resorption were moderately higher than those of the Lean rats. Histopathologically, crown caries progressed to apical periodontitis in $20 \%$ and $50 \%$ of the ZF and ZDF rats' molars, respectively, corresponding to the alveolar bone resorption on the soft X-ray film.
\end{abstract}

Dyslipidemia and hyperglycemia may involve dental caries development and obesity or diabetes could enable lesion progression to apical periodontitis in Zucker rats; diabetes enhances dental caries to a greater extent than obesity.

Keywords: Dental caries; Periodontitis; Obesity; Dyslipidemia; Diabetes; Hyperglycemia; Zucker rat

\section{Introduction}

Several recent epidemiological studies suggested that obesity is an enhancing factor for dental caries [1]. In experimental study, obese and non-diabetic Zucker rats reportedly develop rampant caries involving the entire crown [2].

Meanwhile, numerous conflicting discussions have occurred regarding the causal relationship between dental caries and diabetes in humans because there are many influencing factors associated with dental caries, such as oral hygiene status, age, sex, or any combination of these [3-8]. However, we previously reported that the frequency and severity of dental caries were significantly higher in spontaneous or chemically induced diabetic animal models than those of nondiabetic animals of the same strain and indicated that diabetes is also an enhancing factor for dental caries [7,9].

Both obesity and diabetes are considered to be involved in the development of dental caries that are also characteristics of type 2 diabetes. In $d b / d b$ mice, an animal model of type 2 diabetes was created and demonstrated that the frequency and severity of dental caries were increased compared with that of non-diabetic $d b /+$ mice [10]. However, it remains unclear how obesity and diabetes influence dental caries development and to what extent each factor possesses caries-forming ability.

A Zucker diabetic fatty (ZDF) rat is a strain obtained by selectively mating individual obese Zucker fatty (ZF) rats that developed diabetes and was established as a type 2 diabetes model, which develops diabetes with obesity [11]. Thus, we hypothesized that dental caries would be exacerbated by the addition of the diabetic factor in ZDF rats that have both obesity and diabetes compared to that of obese ZF rats and performed a comparative analysis on the differences in dental lesions among Zucker strains.

\section{Materials and Methods}

\section{Experimental animals}

The rats were handled according to the principles outlined in the Guide for the Care and Use of Laboratory Animals (Setsunan University, Osaka, Japan) and the Japanese Association for Laboratory Animal Science. Six obese or diabetic male ZDF rats aged 40 weeks, along with 8 age-matched ZF and 7 age-matched lean male Zucker (Lean) rats, were used and supplied by Charles River Laboratories

*Corresponding author: Tetsuro Matsuura, Department of Pathology, Faculty of Pharmaceutical Sciences, Setsunan University, 45-1 Nagaotoge-cho, Hirakata Osaka 573-0101, Japan, Tel: +81-72-866-3163; Fax: +81-72-866-3249; E-mail matsuura@pharm.setsunan.ac.jp

Received December 27, 2013; Accepted February 22, 2014; Published February 27,2014

Citation: Kodama Y, Ozaki K, Sano T, Terayama Y, Nishimoto T, et al. (2014) Are Dyslipidemia Following Obesity and Hyperglycemia as an Indicator of Diabetes Involved in Dental Caries Development in Zucker Rats? J Diabetes Metab 5: 340 doi:10.4172/2155-6156.1000340

Copyright: (c) 2014 Kodama Y, et al. This is an open-access article distributed under the terms of the Creative Commons Attribution License, which permits unrestricted use, distribution, and reproduction in any medium, provided the original author and source are credited. 
Citation: Kodama Y, Ozaki K, Sano T, Terayama Y, Nishimoto T, et al. (2014) Are Dyslipidemia Following Obesity and Hyperglycemia as an Indicator of Diabetes Involved in Dental Caries Development in Zucker Rats? J Diabetes Metab 5: 340. doi:10.4172/2155-6156.1000340

Page 2 of 6

Japan, Inc. (Yokohama, Japan). The study rats were housed in stainless steel cages in an air-conditioned animal room $\left(20^{\circ} \mathrm{C}-26^{\circ} \mathrm{C}\right.$; relative humidity, 40\%-70\%) ventilated with filtered fresh air under a 12:12-h light-dark cycle. They were allowed free access to tap water and a widely used standard pellet diet that provided a far lower level of sugar than the established cariogenic diets [12].

\section{Measurement of blood lipid and glucose levels}

Blood triglyceride and total cholesterol levels were measured using a commercial kit (Wako Pure Chemical Industries, Osaka, Japan). The blood glucose level was measured semi-quantitatively by the glucose oxidase method (Glutest Pro R, Sanwakagaku, Aichi, Japan). These were measured when the rats were 13,23, and 33 weeks of age. Blood samples from the tail vein were collected in the afternoon between 1:00 pm and 4:00 pm.

\section{Grading of caries and alveolar bone resorption by soft X-ray examination}

The animals were euthanized by exsanguination under deep anesthesia at the end of the observation period. The mandible and maxilla were removed and fixed in $10 \%$ neutral-buffered formalin (pH 7.4) for $24 \mathrm{~h}$, after which the occlusal, buccolingual, and proximal surfaces of all teeth were examined under a binocular stereoscope. Using fixed samples, soft X-ray photographs of the mesiodistal plane were obtained using $35 \mathrm{kV}$ and $2 \mathrm{~mA}$ for 4 $\min$. The caries scores of each tooth were classified into 4 groups on the basis of the observations and measurements from those photographs: grade 0 , no radiolucent change; 1 , radiolucent area only on the occlusal surface of the crown; 2, radiolucent areas on both occlusal surfaces and either of the mesiodistal surfaces of the crown; 3, radiolucent areas over the entire surface of the crown; and 4, radiolucent areas over almost the entire surface of the dental root. Alveolar bone loss of each tooth was also scored by measuring the radiolucent area around each molar root on the soft X-ray film: grade 0 , no radiolucent change; $1,0.01-0.20 \mathrm{~mm}^{2} ; 2,0.20-0.40 \mathrm{~mm}^{2}$; $3,0.41-0.60 \mathrm{~mm}^{2}$, and $4,>0.61 \mathrm{~mm}^{2}$.

\section{Histopathologic examination}

The right mandible and maxilla of 5 rats from each group were fixed with $10 \%$ neutral buffered formalin for histopathological examination and were decalcified in a $5 \%$ solution of ethylenediaminetetraacetic acid $4 \mathrm{Na}$ (EDTA $4 \mathrm{Na}$ ) for 2 weeks at $4^{\circ} \mathrm{C}$ after fixation. The fixed specimens were trimmed, dehydrated in a sequential ethanol series by an automated processor, and embedded in paraffin wax. Serial 7- $\mu \mathrm{m}-$ thick mesial and distal tooth surface sections were made through the centers of all molars. These sections were stained with hematoxylin and eosin for examination.

\section{Statistical analysis}

The Wilcoxon rank-sum test was employed to compare the differences in the mean scores of the caries lesions and alveolar bone resorption by soft X-ray examination among the groups. The $\chi^{2}$ test was used to determine the incidence of histopathologic lesions, as determined by the histological examinations, in each group. A P value of less than 0.05 was regarded as statistically significant.

The Pearson's correlations were used to examine the associations between molar caries and blood biochemical values (blood glucose, triglyceride, and total cholesterol).

\section{Results}

\section{Body weights}

The average body weights of the ZF group were significantly higher than those of the ZDF and Lean groups at any point. However, those of the ZDF group were comparable to those of the Lean group, and no significant differences existed among them (Figure 1a).

\section{Blood triglyceride and total cholesterol levels}

The average blood triglyceride levels and total cholesterol levels in the ZF group were the highest among the 3 groups, and the difference from that of the Lean group was statistically significant at any point. Each level of the ZDF group showed an upward tendency compared with that of the Lean group, but that was less than half of the ZF group (Figures $1 \mathrm{~b}$ and $1 \mathrm{c}$ ).
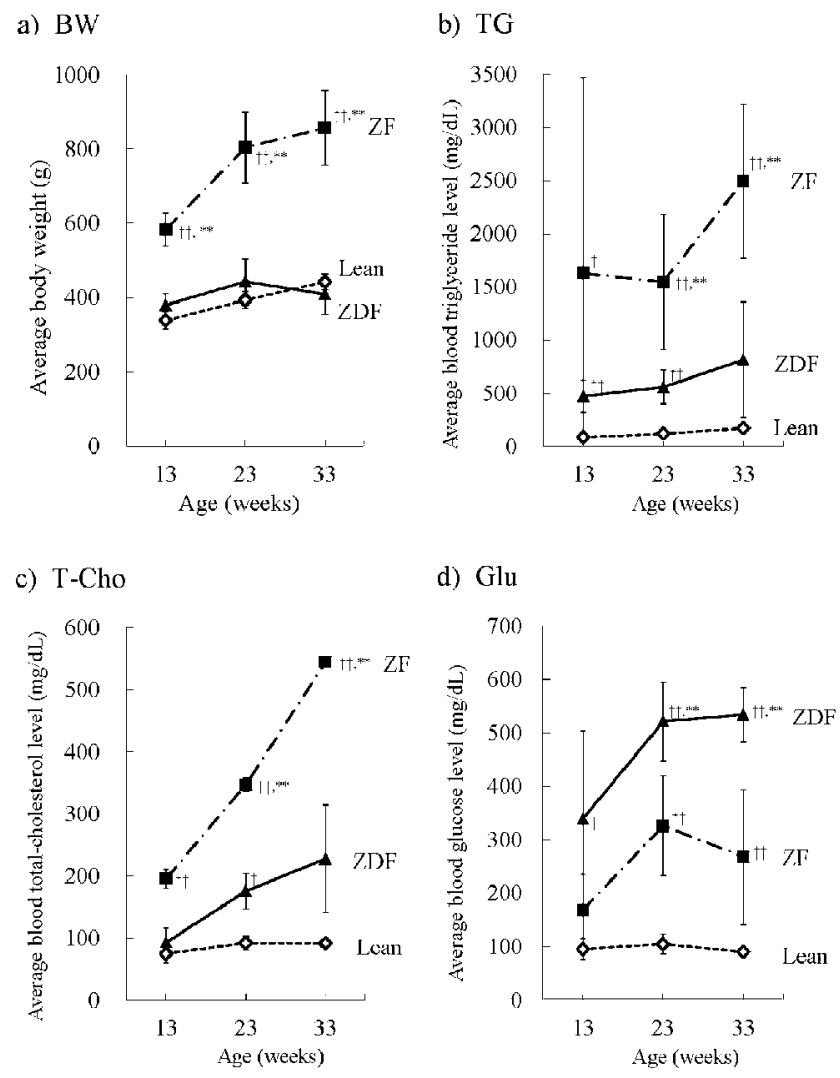

Figure 1: Aging variations regarding body weight, blood lipid, and blood glucose levels

(a) Average body weight changes in the Lean, ZF, and ZDF rats. Significant differences existed between the Lean rats and $Z F$ rats $(\dagger \dagger: P<0.01)$ and between the $Z F$ rats and $Z D F$ rats (**: $P<0.01$ ). (b) Average blood triglyceride level changes in the Lean, ZF, and ZDF rats. Significant differences existed between the Lean rats and $Z F$ rats or between the Lean rats and ZDF rats ( $t: P<0.05$; $\dagger+: P<0.01$, respectively), as well as between the $Z F$ rats and ZDF rats (**: $P<0.01)$. (c) Average blood total cholesterol level changes in the Lean, ZF, and ZDF rats. Significant differences existed between the Lean rats and $Z F$ rats or between the Lean rats and $Z D F$ rats $(\dagger: P<0.05 ; \dagger \dagger: P<0.01$ respectively), as well as between the $Z F$ rats and ZDF rats $\left({ }^{* *}: P<0.01\right)$. (d) Average blood glucose level changes in the Lean, ZF, and ZDF rats. Significant differences existed between the Lean rats and $Z F$ rats or between the Lean rats and $Z D F$ rats $(\dagger: P<0.05 ; \dagger \dagger: P<0.01$, respectively), as well as between the $Z F$ rats and $Z D F$ rats $\left({ }^{* *}: P<0.01\right)$. 
Citation: Kodama Y, Ozaki K, Sano T, Terayama Y, Nishimoto T, et al. (2014) Are Dyslipidemia Following Obesity and Hyperglycemia as an Indicator of Diabetes Involved in Dental Caries Development in Zucker Rats? J Diabetes Metab 5: 340. doi:10.4172/2155-6156.1000340

Page 3 of 6

\section{Blood glucose levels}

The average blood glucose levels of the ZDF group exceeded 500 $\mathrm{mg} / \mathrm{dL}$ at the final point and were significantly higher than those of the ZF and Lean groups at 23 and 33 weeks of age. The level of the ZF group was nearly in the middle of the other 2 strains, ZDF and Lean, and was significantly higher than that of the Lean group at 23 and 33 weeks of age (Figure 1d).

\section{Caries scores by soft X-ray examination}

The macroscopic appearance and soft X-ray photographs of carious mandibular molars in representative cases are shown in Figure 2.

The incidence of molar caries and the mean caries scores in the mandibular and maxillary molars is shown in Table 1 and Figure $3 \mathrm{a}$. Molar caries were detected in both the mandible and maxilla in all groups. In the Lean group, $69.0 \%$ and $2.4 \%$ of the mandibular and maxillary molars, respectively, were affected with dental caries, and the mean caries scores were 1.02 and 0.02 , respectively. Meanwhile, in the ZF rats' mandibular and maxillary molars, the incidence $(93.8 \%$ and $47.9 \%$, respectively) and mean caries scores (1.40 and 0.56 , respectively) were slightly increased compared with those of the Lean group, and there was a significant difference in maxillary caries scores between the 2 groups. Furthermore, in the ZDF group, the incidence (97.2\%) and mean caries score (3.19) in the mandibular molars were significantly higher than those of both the ZF and Lean groups. In the maxilla, a greater number of grade 4 lesions were demonstrated only in the ZDF group, but the mean caries score (0.94) was not statistically significant from that of the ZF group (0.56).

\section{Change in alveolar bone resorption by soft $\mathrm{X}$-ray examination}

The incidence of alveolar bone resorption (ABR) and the mean ABR in the mandibular and maxillary molars is shown in Table 2 and Figure
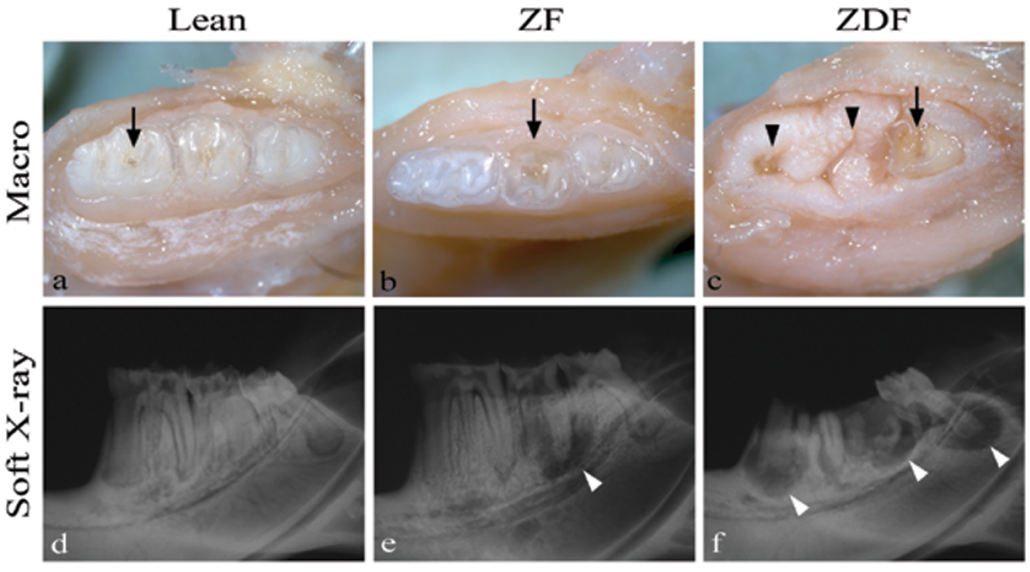

Figure 2: Morphological characteristics of the molar caries in the rats' mandibles.

(a-c) Macroscopic appearances of mandibular molars. (a) A partial coronal defect (arrow) of a first molar (M1) in a Lean rat. (b) A partial coronal defect (arrow) of a second molar (M2) in a ZF rat. (c) A complete coronal defect (arrowheads) of a M1 and M2, and a partial coronal defect (arrow) of a third molar (M3) in a ZDF rat. (d-f) A soft X-ray photograph of the mandible. (d) The parts that correspond with the photo in (a); the radiolucent area in the alveolar bone is not observed. (e) The parts that correspond with the photo in (b); the radiolucent area is observed around the dental root of M2 (arrowhead). (f) The parts that correspond with the photo in (c); the radiolucent area expands extensively around the dental root in the periodontal tissues (arrowheads).

Table 1: Grading and incidence of molar caries in molars.

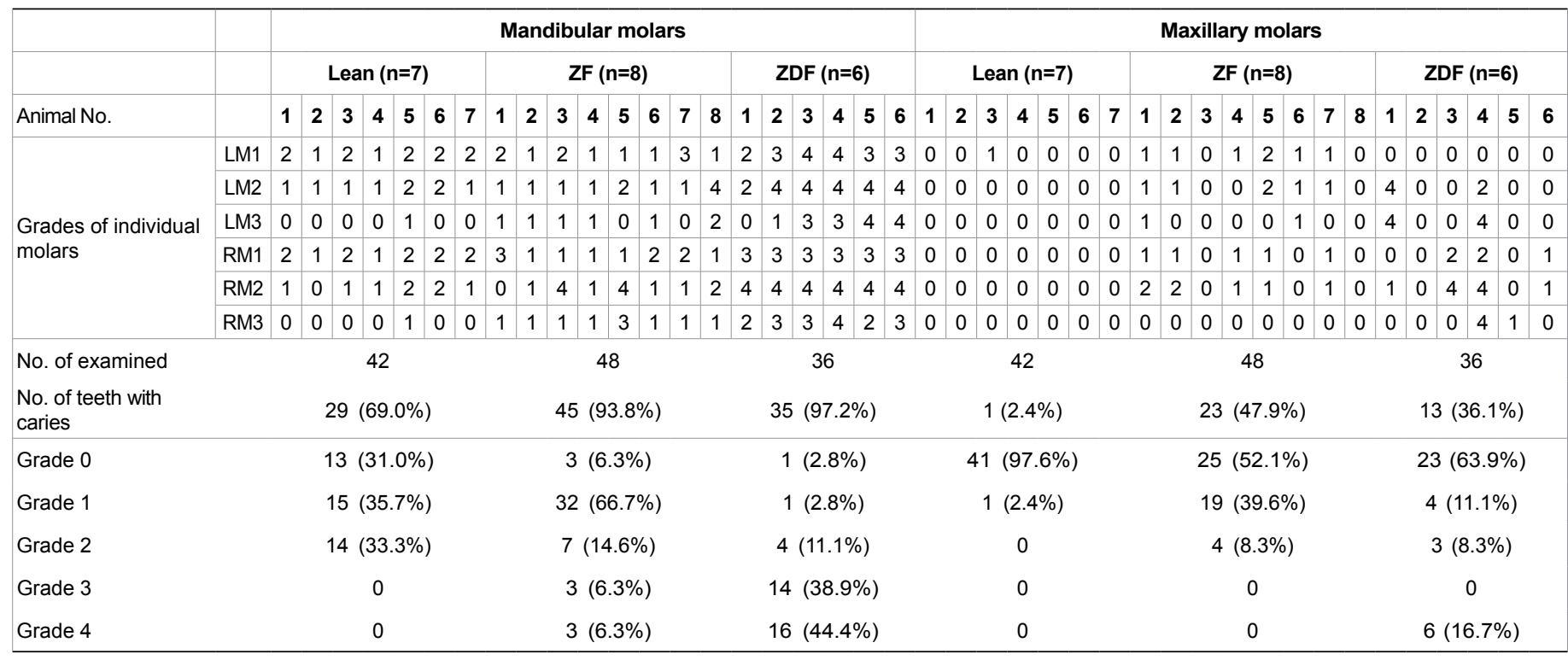


Citation: Kodama Y, Ozaki K, Sano T, Terayama Y, Nishimoto T, et al. (2014) Are Dyslipidemia Following Obesity and Hyperglycemia as an Indicator of Diabetes Involved in Dental Caries Development in Zucker Rats? J Diabetes Metab 5: 340. doi:10.4172/2155-6156.1000340

Page 4 of 6

3b. Alveolar bone resorption was mainly detected in the ZF and ZDF groups. In the Lean group, only $2.4 \%$ of the mandibular molars were affected, and the mean ABR scores were 0.02. In the maxillary molars, no alveolar bone resorption was detected. Meanwhile, in the ZF group,
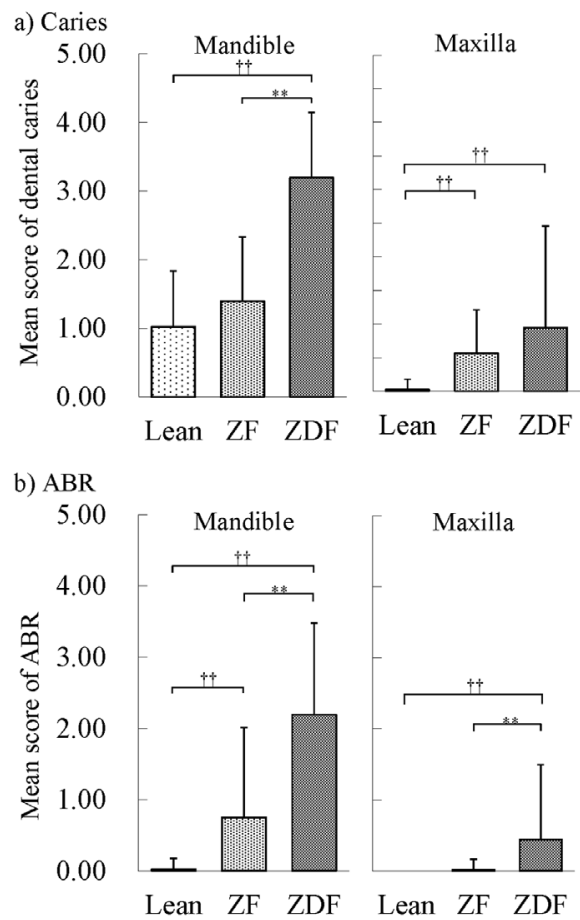

Figure 3: Mean scores of the caries and alveolar bone resorption (ABR) (a) Mean caries score in the mandibular and maxillary molars. A significant difference existed between the Lean rats and $Z F$ rats or between the Lean rats and ZDF rats ( $†+: P<0.01$ ), as well as between the $Z F$ rats and ZDF rats (** $P<0.01$ ). (b) The ABR mean score of the mandible and maxilla. A significan difference existed between the Lean rats and ZF rats or between the Lean rats and $Z D F$ rats $(\dagger+: P<0.01)$, as well as between the $Z F$ rats and $Z D F$ rats $(* *: P<0.01)$
$29.2 \%$ of the mandibular molars and $2.1 \%$ of the maxillary molars were affected, and the mean bone resorption scores for the mandibular and maxillary molars were 0.75 and 0.02 , respectively; the ZF group's mean ABR score for the mandibular molars was significantly higher $(\mathrm{P}<0.01)$ than that of the Lean group. Furthermore, in the ZDF group, $88.9 \%$ of the mandibular molars and $19.4 \%$ of the maxillary molars were affected, and the mean ABR scores (mandibular, 2.19; maxillary, 0.44) were significantly higher $(\mathrm{P}<0.01)$ than those of the other 2 groups.

\section{Histopathological findings}

The incidence of dental and periodontal lesions is summarized in Table 3. Histopathologically, dental caries progressed in the following manner in this study. During the early stage, caries were confined exclusively to the dental crown (Figure 4a), and only crown caries developed without remarkable infiltrative changes in the dental pulp or dental root (Figure 4d). At a more advanced stage, in addition to having crown caries, the lesion penetrated the dentin surface into the dental pulp, with bacterial colonization and neutrophil infiltration, and was considered to be pulpitis accompanied by a partially (Figure $4 \mathrm{~b}$ ) or completely decayed crown (Figure 4c). Furthermore, in almost all cases with pulpitis, the pulpitis expanded into the surrounding dental root through the apical foramen and formed apical periodontitis (Figures $4 \mathrm{e}$ and $4 \mathrm{f}$ ). In the Lean group, the incidence of dental caries was $30.0 \%(9 / 30)$, and the crown caries progressed to dental pulpitis in only 3 cases $(10.0 \%, 3 / 30)$. No apical periodontitis was observed in this group. Meanwhile, in the ZF group, the incidence of dental caries $(66.7 \%, 20 / 30)$ was significantly higher than that of the Lean group, and the crown caries progressed to apical periodontitis in 6 cases $(20.0 \%)$. Furthermore, in the ZDF group, the incidences of dental pulpitis $(60 \%, 18 / 30)$ and apical periodontitis $(50 \%, 15 / 30)$ were significantly increased compared to those of the other 2 groups, and the dental caries were considered worse. The apical periodontitis was usually accompanied by marginal periodontitis and alveolar bone resorption with widening of the periodontal connective tissue space (Figures $4 \mathrm{e}$ and $4 \mathrm{f}$ ), corresponding to the radiolucent area on the soft X-ray film (Figures 2e and 2f). The incidence of marginal periodontitis was almost consistent with that of apical periodontitis; both were highest in the

Table 2: Grading and incidence of alveolar bone resorption (ABR) in surrounding molar by soft X-ray examination.

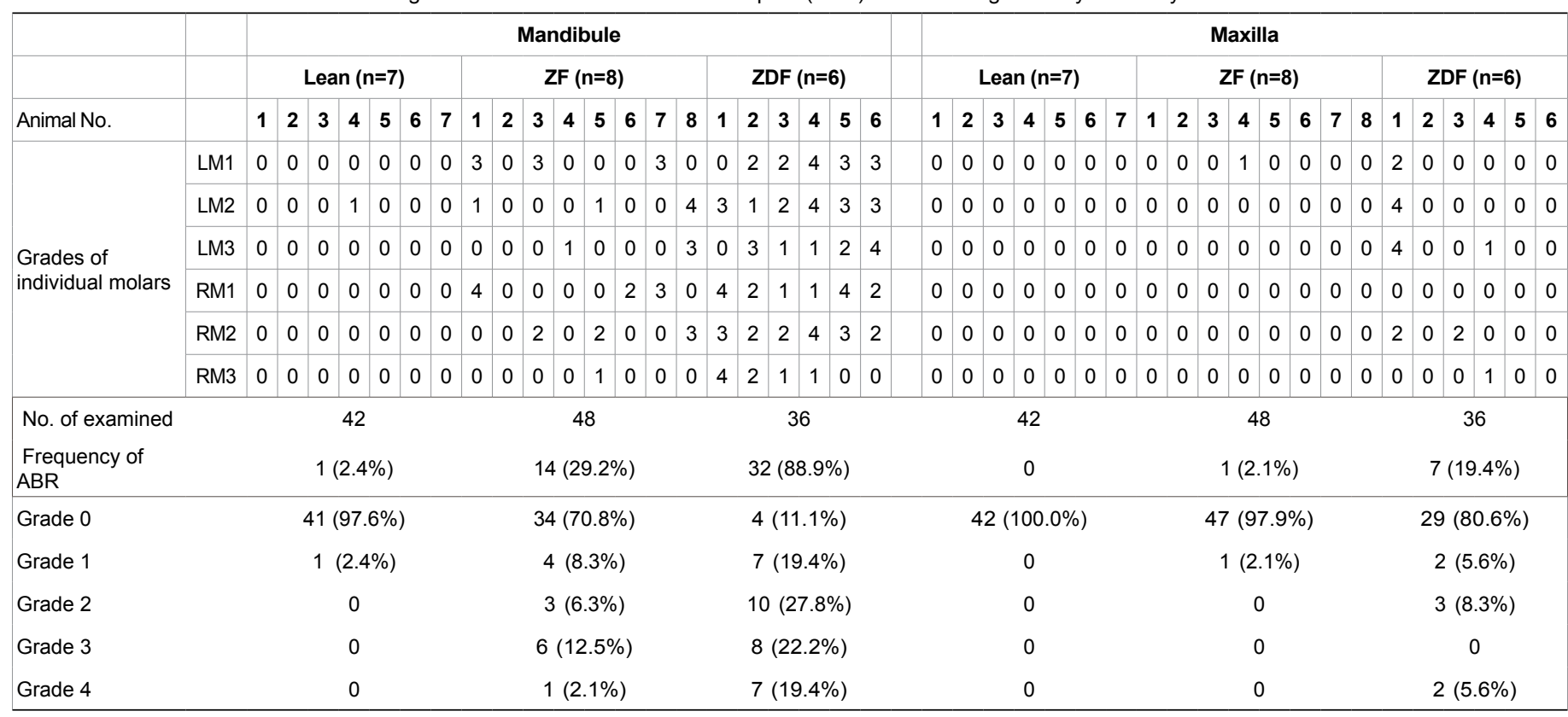


Citation: Kodama Y, Ozaki K, Sano T, Terayama Y, Nishimoto T, et al. (2014) Are Dyslipidemia Following Obesity and Hyperglycemia as an Indicator of Diabetes Involved in Dental Caries Development in Zucker Rats? J Diabetes Metab 5: 340. doi:10.4172/2155-6156.1000340

Page 5 of 6

Table 3: Incidence of histopathologic lesions in the mandibles and maxillae.

\begin{tabular}{|c|c|c|c|}
\hline & Lean $(n=5)$ & $Z F(n=5)$ & ZDF $(n=5)$ \\
\hline Dental caries & $9 / 30(30.0 \%)$ & $20 / 30(66.7 \%)^{\dagger \dagger}$ & $21 / 30(70.0 \%)^{\dagger+}$ \\
\hline Crown caries & $9 / 30(30.0 \%)$ & $20 / 30(66.7 \%)^{\dagger+}$ & $21 / 30(70.0 \%)^{\dagger+}$ \\
\hline Dental pulpitis with crown caries & $3 / 30(10.0 \%)$ & $8 / 30(26.7 \%)$ & $18 / 30(60.0 \%)^{\dagger+, * *}$ \\
\hline $\begin{array}{l}\text { Apical periodontitis with crown } \\
\text { caries }\end{array}$ & $0 / 30$ & $6 / 30(20.0 \%)^{\dagger \dagger}$ & $15 / 30(50.0 \%)^{\dagger \dagger, *}$ \\
\hline Marginal periodontitis & $0 / 30$ & $5 / 30(16.7 \%)^{\dagger}$ & $14 / 30(46.7 \%)^{\dagger+, *}$ \\
\hline Alveolar bone resorption & $0 / 30$ & $6 / 30(20.0 \%)^{\dagger+}$ & $15 / 30(50.0 \%)^{\dagger+, *}$ \\
\hline
\end{tabular}

$t, t+$ significant difference between the Lean and ZF or between the Lean and ZDF $(p<0.05, p<0.01)$.

significant difference between the ZF and ZDF $(p<0.05, p<0.01)$

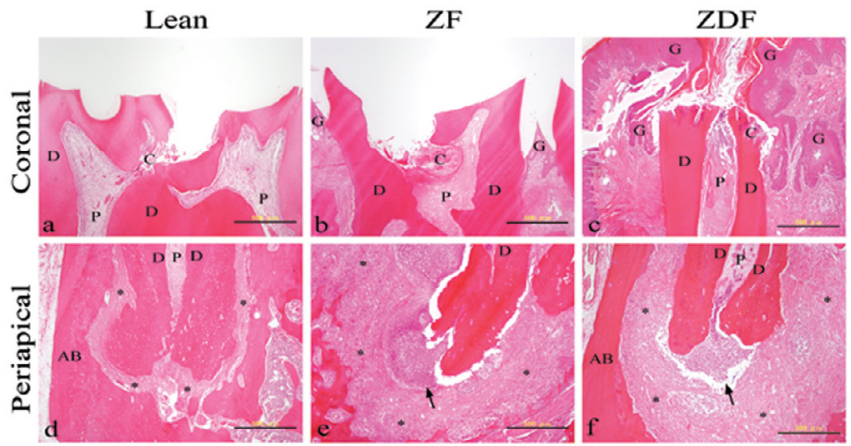

Figure 4: Histopathologic features of molar caries and alveolar bone resorption $\mathrm{AB}$ : alveolar bone; C: dental caries; D: dentin; G: gingiva; P: dental pulp. (a and d) A Lean rat's mandible corresponding to that of the M1 molar area in Figure $2 a$ and $2 d$. (b and e) A ZF rat's mandible corresponding to that of the $\mathrm{M} 2$ molar area in Figure $2 \mathrm{~b}$ and $2 \mathrm{e}$. Inflamed periodontal connective tissue (*) is observed. Note the abscess in the apical part of the tooth (arrow) adjacent to the pulpal inflammation. (c and f) A ZDF rat's mandible corresponding to that of the M1 molar area in Figure $2 \mathrm{c}$ and $2 \mathrm{f}$. Granulation tissue $\left(^{*}\right)$ contains inflammatory cells and epithelial cord with a downward elongation from the gingival surface along the dental root. Note the abscess in the apical part of the tooth (arrow) adjacent to the pulpal inflammation. Bar, $500 \mu \mathrm{m}$.

ZDF group, followed by the ZF group, and were significantly higher $(\mathrm{p}<0.01)$ than those of the Lean group.

\section{Associations between molar caries and blood biochemical value}

Using ZF- and Lean-strain rats, with the exception of the most recently developed ZDF-strain rats, there were similar high positive correlations between caries scores and blood biochemical values (glucose: $\mathrm{r}=0.59, \mathrm{p}<0.05$; triglyceride: $\mathrm{r}=0.63, \mathrm{p}<0.05$; total cholesterol: $\mathrm{r}=0.68, \mathrm{p}<0.01$ ). In all 3 strains, including ZDF-strain rats, there were similar high positive correlations between the incidence of caries (numbers of teeth with caries) and each blood biochemical value (glucose: $\mathrm{r}=0.52, \mathrm{p}<0.05$; triglyceride: $\mathrm{r}=0.52, \mathrm{p}<0.05$; total cholesterol: $\mathrm{r}=0.58, \mathrm{p}<0.01)$. There was a markedly high positive correlation between caries scores and blood glucose $(\mathrm{r}=0.81, \mathrm{p}<0.001)$; however, caries scores correlated poorly with blood lipids (triglyceride: $r=-0.03$; total cholesterol: $\mathrm{r}=0.02$ )

\section{Discussion}

The present study reproduced the presence of dental caries in ZF rat strains, as in a previous report [2]. However, the intensity of the lesions was markedly lower than that of the ZDF group, despite the fact that these rats had obesity characteristics such as being overweight; furthermore, the subsequent dyslipidemia in the ZDF rats was markedly suppressed compared with that of the highly obese ZF strain. In humans, one cannot predict dental caries on the basis of only obesity, but the combination of obesity and an earlier caries experience are more sensitive predictors of future caries than that of an early caries experience alone in children [13]. In addition, normal weight children have significantly fewer dental caries in both their primary and permanent teeth than overweight children [14]. These facts suggest that obesity might contribute to dental caries development, but the cariesforming ability of obesity is weak.

Meanwhile, based on clinical observations, several recent studies suggested that diabetic patients' teeth are predisposed to dental caries $[4,5,15]$. In experimental animals, our previous studies revealed that dental caries are induced in rodents with longstanding diabetes [7]. Furthermore, we reported that alloxan-induced severe hyperglycemia causes rapid-onset and progressive dental caries followed by apical periodontitis in non-obese rats, suggesting that diabetes could independently produce dental caries irrespective of obesity [9]. In the present study, the highly diabetic ZDF rats' dental caries were considerably progressive and enhanced, irrespective of the fact that the rats were less obese compared with that of highly obese and mildly diabetic ZF rats. Thus, it is possible that diabetes possesses potent caries-forming ability and may play a major role in the progression of dental caries in Zucker strains.

The present study also demonstrated new facts including the following: crown caries had already developed in the Lean group, and the lesion progressed to apical periodontitis in the ZF and ZDF groups. Dental caries are not usually induced without cariogenic diets in any experimental animal models including rats, and the CRF-1 diet used in this study provided a lower sugar level that has not previously enhanced dental caries in general rat strains [12]. Therefore, it is likely that the Zucker Lean is a caries-susceptible strain, similar to that of the other diabetic animal models $[7,10]$, and this characteristic might be exacerbated by combining the cariogenic effects of obesity and diabetes in the ZF and ZDF strains. It is also possible that mild hyperglycemia has an effect on the progression of dental caries in the obese ZF strain, but the accurate, independent cariogenic effects of obesity or diabetes still remain unknown in this study.

In conclusion, dyslipidemia and hyperglycemia may involve dental caries development, and obesity or diabetes could enable crown caries to progress to apical periodontitis in Zucker-strain rats. The effects on dental caries are unequal, and diabetes enhances dental caries to a greater extent than obesity.

\section{Acknowledgements}

We would like to thank Ms Michika Kawabe for creating the tissue samples and soft X-ray photographs and for assisting with the morphometrical analysis.

\section{References}

1. Mathus-Vliegen EM, Nikkel D, Brand HS (2007) Oral aspects of obesity. Int Dent J 57: 249-256.

2. Mozaffari MS, Abdelsayed R, Zakhary I, El-Salanty M, Liu JY, et al. (2011) Submandibular gland and caries susceptibility in the obese Zucker rat. J Oral Pathol Med 40: 194-200.

3. Bakhshandeh S, Murtomaa H, Vehkalahti MM, Mofid R, Suomalainen K (2008) Dental findings in diabetic adults. Caries Res 42: 14-18.

4. Miralles L, Silvestre FJ, Hernández-Mijares A, Bautista D, Llambes F, et al. (2006) Dental caries in type 1 diabetics: influence of systemic factors of the disease upon the development of dental caries. Med Oral Patol Oral Cir Bucal 11: E256-260.

5. Twetman S, Johansson I, Birkhed D, Nederfors T (2002) Caries incidence in young type 1 diabetes mellitus patients in relation to metabolic control and caries-associated risk factors. Caries Res 36: 31-35. 
Citation: Kodama Y, Ozaki K, Sano T, Terayama Y, Nishimoto T, et al. (2014) Are Dyslipidemia Following Obesity and Hyperglycemia as an Indicator of Diabetes Involved in Dental Caries Development in Zucker Rats? J Diabetes Metab 5: 340. doi:10.4172/2155-6156.1000340

Page 6 of 6

6. Canepari P, Zerman N, Cavalleri G (1994) Lack of correlation between salivary Streptococcus mutans and lactobacilli counts and caries in IDDM children. Minerva Stomatol 43: 501-505.

7. Kodama Y, Matsuura M, Sano T, Nakahara Y, Ozaki K, et al. (2011) Diabetes enhances dental caries and apical periodontitis in caries-susceptible WBN/ KobSlc rats. Comp Med 61: 53-59.

8. Tagelsir A, Cauwels R, van Aken S, Vanobbergen J, Martens LC (2011) Dental caries and dental care level (restorative index) in children with diabetes mellitus type 1. Int J Paediatr Dent 21: 13-22.

9. Nakahara Y, Sano T, Kodama Y, Ozaki K, Matsuura T (2012) Alloxan-induced hyperglycemia causes rapid-onset and progressive dental caries and periodontitis in F344 rats. Histol Histopathol 27: 1297-1306.

10. Sano T, Matsuura T, Ozaki K, Narama I (2011) Dental caries and caries-related periodontitis in type 2 diabetic mice. Vet Pathol 48: 506-512.
11. Clark JB, Palmer CJ, Shaw WN (1983) The diabetic Zucker fatty rat. Proc Soc Exp Biol Med 173: 68-75

12. Fukuzato $\mathrm{Y}$, Matsuura T, Ozaki K, Matsuura M, Sano T, et al. (2009) Morphological study on dental caries induced in WBN/KobSlc rats (Rattus norvegicus) fed a standard laboratory diet. Lab Anim 43: 376-381.

13. Tuomi T (1989) Pilot study on obesity in caries prediction. Community Dent Oral Epidemiol 17: 289-291.

14. Willershausen B, Haas G, Krummenauer F, Hohenfellner K (2004) Relationship between high weight and caries frequency in German elementary schoo children. Eur J Med Res 9: 400-404.

15. Taylor GW, Manz MC, Borgnakke WS (2004) Diabetes, periodontal diseases dental caries, and tooth loss: a review of the literature. Compend Contin Educ Dent 25: 179-184, 186-8, 190. 